\title{
Eside
}

\section{Pleural effusion as a late complication of intravesical BCG treatment}

\author{
Ailish Nimmo, ${ }^{1}$ Amy Gray, ${ }^{1}$ Aarti Patel, ${ }^{2}$ Joseph MacKenzie, ${ }^{1}$ Ian Fairbairn ${ }^{1}$
}

${ }^{1}$ Respiratory Department, Victoria Hospital, Kirkcaldy, UK ${ }^{2}$ Histopathology Department, Victoria Hospital, Kirkcaldy, UK

\section{Correspondence to} Dr Ailish Nimmo, Victoria Hospital, Hayfield Road, Kirkcaldy KY2 5AH, UK; ailish. nimmo@googlemail.com

Received 12 June 2017 Revised 4 October 2017 Accepted 9 October 2017 Published Online First 26 October 2017
Amy Gray (Junior Doctor): An 88-year-old man with a background of transitional cell bladder cancer diagnosed in 2012 was referred to the ambulatory care clinic with a 1-month history of increasing breathlessness. He had constitutional symptoms of fatigue, reduced appetite and weight loss for the past 4 months. There was no history of cough, fever or night sweats. His bladder cancer had been resected in 2013 with pathology confirming this as a G3 pT1 transitional cell cancer (TCC), and there was no evidence of recurrence on surveillance flexible cystoscopies. He had been on maintenance intravesical BCG therapy since this time, last given 8 months previously. Of note, his last BCG instillation had been traumatic with haematuria postprocedure. He was an ex-smoker of 25 years and had worked within manufacturing and sales in the wallpaper industry. He had no known exposure to asbestos or TB and no animal exposure.

He had a chest X-ray, which showed a left-sided pleural effusion taking up 50\% of the hemithorax (figure 1). A CT chest was then performed, which confirmed a moderate left-sided pleural effusion and also showed a spiculated $16 \mathrm{~mm}$ nodule in the left upper lobe and a spiculated $23 \mathrm{~mm}$ nodule in the right upper lobe. These were not present on a previous CT in 2014.

Ian Fairbairn (Respiratory Consultant): In summary, this elderly man with a background of bladder TCC presents with a large left-sided pleural effusion and pulmonary nodules. A unilateral effusion in the presence of pulmonary nodules is more likely to be exudative than transudative in nature, with a differential diagnosis including malignant, infective and inflammatory conditions. Bladder TCC can metastasise to the lung and also less frequently to the pleura. ${ }^{1}$ Given his history and

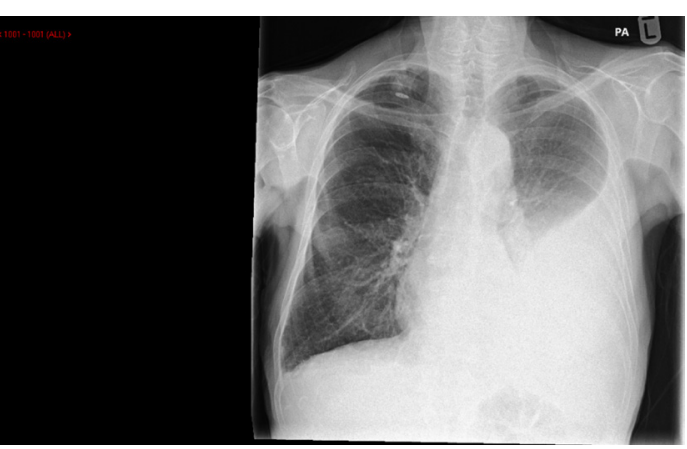

To cite: Nimmo A, Gray A, Patel $A$, et al. Thorax 2018:73:393-394.
Figure 1 Initial chest X-ray showing large left-sided pleural effusion. the CT appearances, a diagnosis of neoplasm was our main concern at this point. Pleural aspiration for biochemistry, cytology and culture is required to differentiate between transudative and exudative effusions and investigate for a malignant or infective cause.

AG (Junior Doctor): Pleural aspiration yielded straw-coloured fluid. Biochemistry revealed this to be an exudate by Light's criteria (pleural lactate dehydrogenase $536 \mathrm{U} / \mathrm{L}$, protein $36 \mathrm{~g} / \mathrm{L}$; serum lactate dehydrogenase $348 \mathrm{U} / \mathrm{L}$, protein $65 \mathrm{~g} / \mathrm{L}$ ). Cytology showed a lymphocyte-rich effusion with no malignant cells. Fluid was also sent for routine and mycobacterial culture, with initial results showing no acid-fast bacilli seen and no growth on routine culture.

Joseph MacKenzie (Respiratory Consultant): The differential diagnosis for a lymphocyte-rich exudative effusion includes neoplasm, mycobacterial infection and inflammatory conditions. Early medical thoracoscopy has the advantage of draining the fluid to relieve breathlessness, obtain pleural biopsies for a definitive diagnosis and enable pleurodesis to prevent reoccurrence. The patient returned to hospital 1 week after his initial presentation to have this procedure performed. At thoracoscopy, the pleura was noted to appear abnormal with adhesions, nodules and areas of leukoplakia. A total of $1.5 \mathrm{~L}$ of fluid was drained. Pleural biopsies were taken for pathology. In view of the man's frailty, the concern of underlying malignancy and risk of reoccurrence of the effusion a talc poudrage was performed at the end of the procedure with the aim of pleurodesis. Postprocedure chest X-ray showed partial expansion of the left lower lobe. The patient reported symptomatic relief and was discharged 4 days following thoracoscopy to await his results.

Aarti Patel (Consultant Histopathologist): Pleural biopsies from thoracoscopy returned showing dense infiltrates of histiocytes and multinucleated cells with focally necrotising granulomas (figure 2). Staining including Ziehl-Neelsen (TB), Wade Fite (Mycobacterium leprae), periodic acid-Schiff (adenocarcinoma) and Grocott (fungal infection) was negative. Necrotising granulomas are highly suggestive of mycobacterial infection, of which Mycobacterium tuberculosis is by far the the most common to infect the pleura. BCG is far less pathogenic but can cause a disseminated infection postbladder instillation associated with non-caseating granulomas in the lung on transbronchial biopsy. ${ }^{2}$ At the time of pathology return, there was 


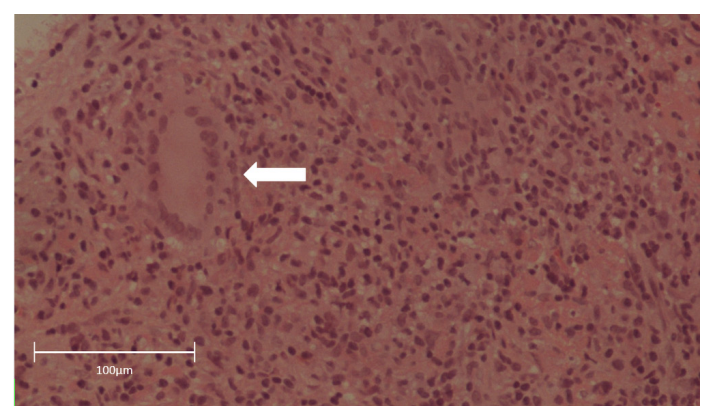

Figure 2 Pleural biopsy demonstrating pleural inflammation with focally necrotising granuloma.

no growth on pleural fluid routine and mycobacterial culture. Standard TB quadruple therapy with rifampicin, isoniazid, pyrazinamide and ethambutol was considered but not immediately commenced as the patient was well.

Ailish Nimmo (Junior Doctor): One week after the return of the pleural biopsy report and prior to quadruple therapy being commenced, mycobacterium was cultured from the initial pleural fluid sample. This was subsequently identified as Mycobacterium bovis BCG strain sensitive to ethambutol, isoniazid and rifampicin and resistant to pyrazinamide. The patient was well and commenced on dual therapy with rifampicin and isoniazid.

Three days after commencing therapy, the patient represented with increasing breathlessness, malaise and cough. Chest X-ray showed reaccumulation of the left pleural effusion and increased right upper lobe opacification. Secondary bacterial infection, immune hypersensitivity and failure of treatment were all considered. Sputum culture grew respiratory commensals only, making the latter two options more likely. He was therefore commenced on ethambutol and levofloxacin in addition to rifampicin and isoniazid (based on sensitivity results), and $60 \mathrm{mg}$ prednisolone was added. Over the next week, his symptoms improved and he was discharged home on the same antibiotics and a reducing course of prednisolone.

The patient's progress has been complicated by drug-induced hepatitis resulting in cessation and subsequent reintroduction of his medications. His steroids were weaned and his antibiotics rationalised to rifampicin, isoniazid and ethambutol with a plan to continue for 9 months in total given his disseminated culture-positive disease. He remains well with no reoccurrence of his breathlessness and a stable small loculated left pleural effusion 8 months after starting treatment.

IF (Respiratory Consultant): BCG is a live attenuated strain of $M$. bovis with reduced pathogenicity. In superficial bladder cancer, BCG activates the cell-mediated immune system, which targets an attack on malignant cells. The exact intravesical BCG regimen varies depending on the risk of disease recurrence, but typically involves maintenance therapy being given at 3-6 monthly intervals for up to 3 years following surgical resection.

Intravesical BCG frequently causes mild and self-limiting side effects, including localised symptoms such as dysuria and haematuria in up to $70 \%$ of individuals and systemic symptoms such as fever in up to $25 \%$. Serious complications are seen in a much smaller proportion of patients, and disseminated BCG infection is reported in $0.4 \%$ of cases. ${ }^{3}$ It is not clear what leads some immunocompetent individuals to develop severe systemic complications over others. Traumatic instillation of BCG does seem to be associated with increased complications due to loss of the urothelial barrier allowing access of the organism to the bloodstream. Treatment is also recommended to be avoided in cases of gross haematuria, recent biopsy or resection of the bladder or prostate, or states of immunocompromise. ${ }^{4}$

BCG complications are thought to be due to active infection, a hypersensitivity reaction or a combination of both. The difficulty of culturing BCG mycobacterium and improvement in some patients after glucocorticoid treatment initially supported the hypersensitivity hypothesis. ${ }^{5}$ However, organisms have been identified in several different tissues including lung, liver, pancreas and now pleural fluid, suggesting disseminated infection. The fact that complications can develop from months up to years after BCG administration also supports this theory. ${ }^{5}$ Treatment has therefore typically consisted of both glucocorticoids and antimycobacterial therapy.

\section{TAKE HOME MESSAGE}

To the best of our knowledge, this is the first case report of intravesical BCG resulting in pleural necrotising granulomas and culture of the organism in pleural fluid. These features suggest a severe disseminated active infection rather than a hypersensitivity reaction, with the patient requiring aggressive antimicrobial therapy. This case illustrates the need to consider disseminated BCG infection in patients presenting with pleural effusion who have previously received intravesical BCG.

Contributors The authors of this paper, $A N, A G, A P, J M K$ and IF, were all involved in the care of the patient, the interpretation of results, and the writing and revision of the paper and are accountable for the accuracy and integrity of the work.

Competing interests None declared.

\section{Patient consent Obtained.}

Provenance and peer review Not commissioned; externally peer reviewed.

(c) Article author(s) (or their employer(s) unless otherwise stated in the text of the article) 2018. All rights reserved. No commercial use is permitted unless otherwise expressly granted.

\section{REFERENCES}

1 Bonnin N, El Karak F, Droz JP, et al. Pleural metastasis in a patient with bladder cancer. $J$ Clin Oncol 2008;26:329-30.

2 LeMense GP, Strange C. Granulomatous pneumonitis following intravesical BCG. What therapy is needed? Chest 1994;106:1624-6.

3 Koya MP, Simon MA, Soloway MS. Complications of intravesical therapy for urothelial cancer of the bladder. J Urol 2006;175:2004-10.

4 Paterson DL, Patel A. Bacillus Calmette-Guerin (BCG) immunotherapy for bladder cancer: review of complications and their treatment. Aust N Z J Surg 1998;68:340-4

5 Gonzalez OY, Musher DM, Brar I, et al. Spectrum of bacille Calmette-Guérin (BCG) infection after intravesical BCG immunotherapy. Clin Infect Dis 2003;36:140-8. 